\title{
A Personal Communicator of the Vocally Handicapped
}

\author{
Richard Reilly, Student Member, IEEE, Annraoi de Paor \\ Department of Electronic and Electrical Engineering, University College Dublin, \\ Belfield, Dublin 4, Ireland.
}

\begin{abstract}
An affordable personal communicator which allows full control of the voice has been designed and developed. The aid employs coded digitised speech in an efficient manner, coupled with specific hardware in an attempt to address the problems which normally beset such communicators. Speech is recorded, digitised and compressed under ADPCM from 12 to 4-bits. The compressed waveform is transferred into a portable handheld, discreet playback unit, under the control of specific graphic interface software, allowing complete customisation of the handheld unit for the user or application.
\end{abstract}

\section{INTRODUCTION}

In the design of augmentation aids one has to be aware of the disability in question. Speech disorders can be attributable to either mental or physical damage to certain locations within the brain. Most speech disorders are a result of physical damage to the voice production mechanisms in Broca's Area. Therefore, one has to be mindful whether impaired speech or impaired language skills is at issue. Someone with a mental disability which affects their general language ability will never be able to communicate using synthetic speech or through other methods such as sign language.

According to Edwards [1] the most important features in the design of a synthatic voice, besides the quality of the voice, are the speed of response, vocabulary and cost. The design of an augmentation aid is a most demanding project and the most demanding for a synthetic voice. This is so due to the fact that it must replace all the qualities of natural speech for someone unable to speak. It must project an oral personality for someone who is unable to fulfil that role for themselves. In such an assessment, speed is of lower priority than naturalness of speech. Due to this fact, the voice of the synthesizer should match the other components of the user's personality. The availability of commercial devices which employ this reasoning is very limited. Most have a factory-set voice, which normally is of an American male. The appeal of such devices therefore to

This work was supported by the National Medical Rehabilitation Trust
European females is limited.

To be useful in everyday conversation the vocabulary of the aid should be unlimited. This would imply that stored natural speech samples would not be applicable. However the speed of generation of speech is vital, since the user must be able to interrupt into conversation at any time as natural speakers do. With an unlimited vocabulary system this is not possible. Hence the time between requesting speech and its being generated is crucial.

The design of the system-user interface is also critical. It is imperative to reduce the amount of time spent at the interface while maintaining high quality speech. Since a significant number of users suffer from physical conditions that restrict their manual dexterity, the number of actions to generate speech must be kept to a minimum. The generation or selection of speech is normally achieved through location and activation of a switch or key.

The design requirements of speech quality, speed of response, vocabulary and cost, have not all been adhered to in the commercially available devices. It was believed that the inclusion of size in the above list, was imperative if the aid was to be used as a personal communicator. The dimensions of a device were be such that the user could comfortably transport and use it discreetly in conversation.

Considering the available devices and the design specifications laid down by therapeutic staff, a small handheld unit employing digitised speech was conceived. The storage of speech had to be such that the vocabulary could easily be altered or modified as required. The unit was required to store approximately two minutes of speech, with this limit expandable at a later date.

\section{HARDWARE AND SOFTWARE DESIGN}

Following the choice of ADPCM as a compression mechanism and subsequently a suitable processor, MSM5218 [2], hardware circuitry was designed and constructed. A circuit utilising this chip built by Keating et 
$a l$, for their Pocket Speech Aid [3], [4]. This design allowed for 8-bit speech data to be stored, and implanted on EPROM for use in a small communicator. The speech with this system is recorded using a BBC Model B computer. This recording system is inflexible due to hardware constraints and to the use of the Model B computer. The use of 8-bits by Keating reduces memory requirements but also reduces the quality of the resultant speech. The design described has tried to provide a more adaptable system.

The system consists of two separate units. A recording unit centred about a 12-bit ADC [5] and the MSM5218, and the playback unit centred about the $\mu$ PD78C10 microprocessor [6]. The recording system is connected via a peripheral interface adapter to an IBM PC. The software to control the digitisation is presented to the user or therapist in the form of Graphical User Interfaces [7]. This allows for a complete customisation of the playback unit from message inclusion to variable length

The NEC $\mu \mathrm{PD} 78 \mathrm{C} 10$ was chosen due to its availability, programming ease and affordable cost. The single chip 8bit microcomputer's 40 I/0 lines are available for EPROM memory addressing. The recorded speech is compressed from 12 to 4 - bits and then stored in EPROM form within the playback unit.

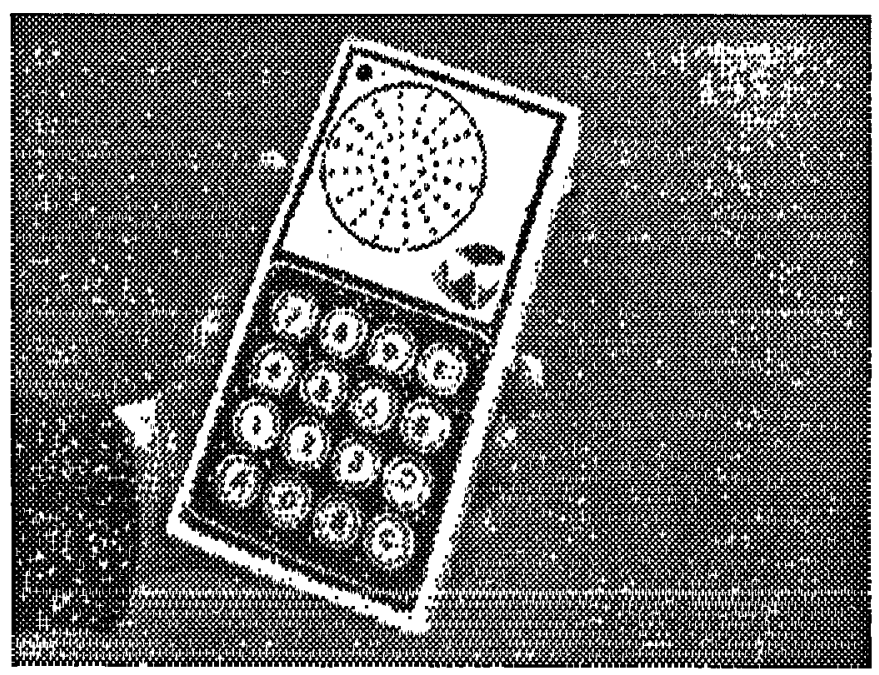

Fig 1. The dimensions of the Playback Unit

One important feature incorporated in the handheld device at this stage, was the microprocessor controlled power supply LM2984C. This allowed the power lines to drop to a standby voltage level during inter-message intervals. This facility increases the battery life by allowing maximum power consumption only during the playback of messages.

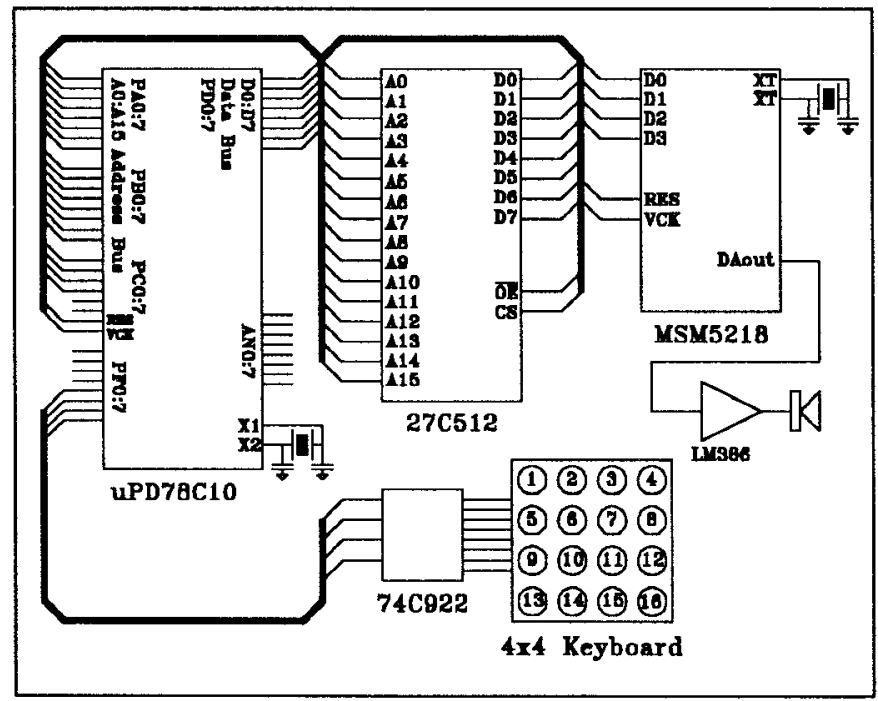

Fig 2. Playback Circuit incorporating $\mu$ PD78C10 and MSM5218

\section{CONCLUSION}

The portable speech playback unit coupled with the fixed recording unit allows for high quality instantaneous speech at an affordable cost.

\section{REFERENCES}

[1]. Edwards, A.D.N. Speech Synthesis in Augmentative Communication. IEE Colloquium on System and Applications of Man-Machine Interaction using Speech I/O. Digest No.1991/06, March 1991.

[2] Oki Semiconductor Oki Voice Synthesis LSI Databook 3rd edition. 785 North Mary Avenue, Sunnyvale, California, USA, 94086., 1990

[3] Keating, D.,Smith, D.C., Evans, A.L., Wyper, D.J., Cunningham, E. "The pocket speech aid". Medical \& Biological Engineering \& Computing, 27, 288-290., 1989

[4] Evans, A.L. \& Fenner, J. "A random access phrase recorder" Electronics \& Wireless World, 92, 54-56., 1987.

[5] Analog Devices Inc : Digital Signal Processing Division, 1 Technology Way, Norwood, Massachusetts, 02062, USA.

[6] NEC Electronics (Europe) GmbH, Oberrather Str. 4, 4000 Düsseldorf 30, Germany.

[7] Reilly R, dePaor A. "A personalised communicator for the vocally handicapped employing waveform coding and graphical user interfaces".submitted to Innovation et Technologie en Biologie et Medecine. 